\title{
Towards Automatic Speaker Retrieval for Large Multimedia Archives
}

\author{
Marijn Huijbregts \\ Radboud University Nijmegen \\ marijn.huijbregts@let.ru.nl
}

\author{
David van Leeuwen \\ Radboud University Nijmegen \\ d.vanleeuwen@let.ru.nl
}

\begin{abstract}
In this paper we discuss the challenges of scaling a speaker retrieval system for small audiovisual collections towards a speaker retrieval system for large audio (visual) archives. We show that with our large scale speaker diarization approach it is possible to perform query-by-example speaker retrieval; to search for audiovisual documents in which a particular person is talking. On a selection of the ICSI meeting corpus we obtain a Mean Average Precision of 0.49 and precisionat-ten of 0.70 . On a much larger archive of three months of Dutch broadcast television we obtain a precision-at-ten of 0.52 .
\end{abstract}

Categories and Subject Descriptors:

J.0 [Computer Applications]: General

General Terms:

Algorithms, Experimentation, Performance

Keywords: Speaker retrieval, speaker diarization, speaker tracking, large scale diarization

\section{INTRODUCTION}

An important source of information for search in audiovisual material is in what is said. For the semantic interpretation and acceptance of a verbal statement it is not only useful knowing what was said, but also who said it. Speaker information makes it possible to search for recordings of specific speakers, but it can also aid in various automatic annotation tasks such as topic boundary detection, summarization or automatic speech recognition.

Although automatic recognition of speakers is investigated in a number of research fields, there does not yet exists a system that is able to fully automatically annotate large multimedia archives with the true identity of each speaker. The requirements of such a speaker retrieval system are very demanding. First, it should not be confused by non-speech sounds, these need to be filtered out. Next, it should be able to segment the speech such that each segment contains speech from a single speaker. Then it needs to be able to

Permission to make digital or hard copies of all or part of this work for personal or classroom use is granted without fee provided that copies are not made or distributed for profit or commercial advantage and that copies bear this notice and the full citation on the first page. To copy otherwise, to republish, to post on servers or to redistribute to lists, requires prior specific permission and/or a fee.

AIEMPro'10, October 29, 2010, Firenze, Italy.

Copyright 2010 ACM 978-1-4503-0164-0/10/10 ...\$10.00. collect all speech that originates from the same speaker into a cluster, not only within each recording but for the entire archive. Finally the system should be able to label each cluster of speaker segments with the identity of the corresponding speaker.

In previous work we have developed a system that is able to perform the first steps of such a full speaker retrieval system on relatively small multimedia collections: segmenting and clustering all speech in the collection on a speaker basis [6]. The system is able to perform speaker diarization (segmentation and clustering) not only for each recording in the collection, but also collection-wide. We therefore refer to this approach as large scale speaker diarization. With the large scale diarization system it is not yet possible to search people using their name, but it is possible to perform queryby-example speaker retrieval. In this kind of retrieval, the user selects a small audio(visual) fragment in which someone is talking and the system then retrieves a list of documents in which the same person is speaking. This is similar to image retrieval where an example image is used as query to find other similar images. For an audiovisual archive, obviously the two techniques can be combined.

In this paper we will discuss our ongoing work towards improving our system so that it is able to perform query-byexample speaker recognition on large archives. We will show that a number of challenges still needs to be solved in order to perform robust speaker retrieval, but that with some adjustments of our existing large scale diarization system it is already possible to perform query-by-example retrieval on an archive of three months of Dutch broadcast television.

The remainder of this paper is organized as follows. First we will discuss existing speaker recognition work. In section 3 we will then describe our approach of speaker retrieval for small multimedia collections and we will demonstrate its applicability on a small collection. In sections 4 and 5 we will introduce a bigger video archive and we will discuss the problems that we encounter in applying our system on this archive.

\section{RELATED WORK}

A number of research fields investigate the task of automatically obtaining speaker information in various ways. In this section we will discuss three of them: speaker diarization, speaker recognition and speaker tracking.

\subsection{Speaker diarization}

The goal of speaker diarization is to automatically segment an audio recording into speaker homogeneous regions, 
and link these together. With the identity of each speaker and even the number of speakers unknown, it is the task of a diarization system to anonymously label each speaker in the recording and answer the question: 'Who spoke when?' [9].

NIST has organized speaker diarization benchmarks for the meeting domain since 2004 [3]. At each benchmark, diarization systems are evaluated on excerpts of meeting recordings. Each recording typically is 15 to 30 minutes long and contains three to ten speakers. Speaker diarization can be regarded as the starting point for full speaker retrieval, but the focus of the NIST benchmarks is not to identify the speakers (label the clusters with names) and does not require of the the systems to scale beyond the small recording excerpts.

Most diarization systems are based on agglomerative clustering techniques where the number of initial clusters depends on the length of the recording. Because the number of needed clustering steps increases linearly with the length $T$ of the recordings and because all combinations of two clusters need to be compared at each clustering step $\left(\mathcal{O}\left(T^{2}\right)\right.$ for each step), the complexity of the standard speaker diarization approach scales with the total duration of speech in the archive $T$ as $\mathcal{O}\left(T^{3}\right)$. With such a complexity it is not straightforward to cluster all speech of all speakers in a large archive.

\subsection{Speaker recognition}

In speaker recognition the basic task is to determine whether or not two segments of speech originate from the same speaker. Often one of the segments, the training segment, is relatively long (up to several minutes of speech) while the other segment, the test segment, typically is a lot shorter (down to a few seconds only).

Speaker recognition on its own is not the solution for creating a speaker retrieval system, but it is a key technique in combination with other approaches such as speaker tracking. A lot of the test-independent speaker recognition system development is carried out in the context of benchmark evaluations, such as regularly organized by NIST since 1996, and recently also in the Italian 'Evalita' campaign in 2009.

\subsubsection{Summed channel conditions}

One reoccurring condition in the NIST evaluations are "summed channel" or "2-wire recording" conditions, where the two speakers of the conversation occur on a single recording channel. The training samples consist of three or eight conversations where the target speaker occurs, which must be found automatically before a model for that speaker can be made. This task has speaker diarization aspects to it, where additional boundary conditions are given.

\subsection{Speaker tracking}

In speaker tracking, the task is to find spoken segments of a particular speaker for which some training material is given $[8,1]$. This has both aspects of diarization, as the audio stream needs to be segmented first, and of operation on a collection of recordings. It has as such been performed in both the 1999/2000 editions of the NIST SRE for 2-wire telephone conversations and the French ESTER 2005 evaluation campaign for broadcast news. The task and evaluation measures were cast in a detection and retrieval framework, respectively, and can be compared to a "known item retrieval" task in text retrieval. Most speaker tracking systems solve the task by performing speaker diarization followed by speaker recognition [10,13]. Although this problem is similar to our definition of a speaker retrieval system, there is an important difference: we would like to be able to retrieve the speech of all speakers in a collection, not just the speech of speakers known a-priory.

\section{LARGE SCALE DIARIZATION}

In our quest to full speaker retrieval, in this paper we will first focus on part of the problem: automatically segmenting and clustering all speech from a collection. This task, that we will call Large Scale Speaker Diarization, is similar to speaker diarization, except that speaker labels should not only be unique within each recording, but across the entire collection. Large scale speaker diarization is also similar to speaker tracking, except that it should be possible to uniquely label every speaker in the collection and not only a set of known speakers.

In this section we will first summarize our method of performing large scale speaker diarization and then discuss a retrieval experiment on a relatively small collection. An in-depth description of our large scale speaker diarization system can be found in [6].

\subsection{Procedure}

The large scale diarization procedure consists of five steps: Speech Activity Detection (SAD), chunking, diarization, enrollment and linking. Figure 1 depicts the procedure.

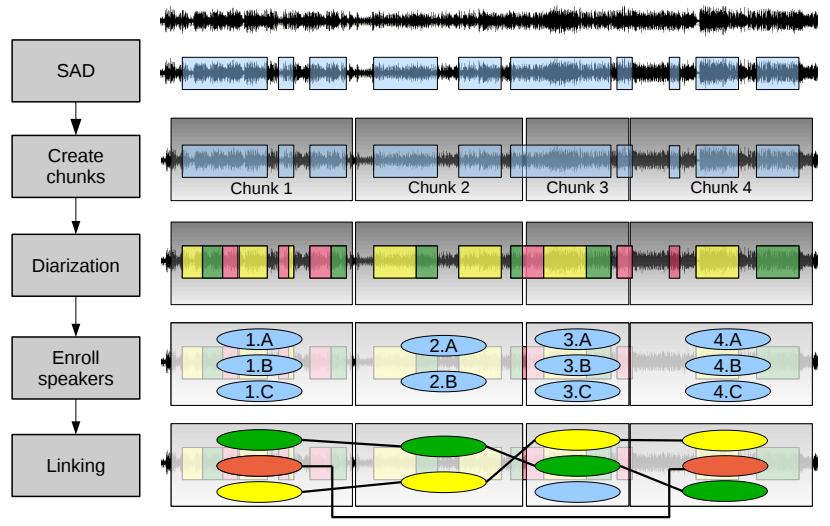

Figure 1: The five steps of our procedure for large scale speaker diarization.

In the first step of the algorithm, all speech regions need to be found and all non-speech fragments need to be discarded. This Speech Activity Detection (SAD) step is especially hard when the audio does not only contain clean speech and silence but also music and sound effects or speech in adverse conditions. In [7] we propose a SAD system that copes with these types of audio. We use this system without adjusting it in any way.

Secondly, the recording is cut-up in evenly sized chunks of duration $T_{c}$. Note that this does not mean that each chunk is of the exact same length; they only contain the same amount of speech. In the third step, speaker diarization is performed on each chunk. For diarization we use the system that we developed for the NIST speaker diarization benchmarks $[12,11]$. Because of the chunking, it does not 
matter if a collection consists of a few very long recordings or of many short recordings. In both cases a similar set of evenly sized chunks are generated.

Fourth, for each speaker cluster that is found by the diarization system, a speaker recognition model is trained (this is called speaker enrollment). After enrollment, the data of all speakers is scored against each speaker model. The resulting matrix of scores is used in the final step to link the speakers across chunks. The speaker recognition system that we use for this step, developed for the Evalita'09 benchmark, is described in [5]. Similarly to SAD and diarization, we use this system without adjusting it in any way.

The matrix with scores of all speaker recognition models on the available data of each speaker will be used to link the speakers across chunks ${ }^{1}$. In linking the speakers, we will apply standard agglomerative clustering with the restriction that speakers from the same chunk are not allowed to be linked. We apply this restriction because we assume that after diarization, each individual chunk contains unique speakers and that the diarization system will not output more than one cluster for each speaker.

Our procedure for large scale diarization is closely related to speaker tracking (see section 2), except that we try to link the speech of all speakers in a collection, not just the speech of a-priory known speakers. In order to do this we do not only apply speaker recognition on (impure) speaker clusters, we also train the speaker recognition models on these clusters. Note that it is possible to combine the speaker tracking and large scale diarization strategies: once a large part of a collection is diarized (across the collection), we could from then on perform speaker tracking on new recordings using the automatically obtained speaker recognition models.

\subsection{Speaker retrieval experiment}

In this section we will discuss an experiment that shows that it is possible to perform query-by-example speaker retrieval on the output of our large scale diarization system. We have performed the experiment on part of the ICSI meeting corpus [2]. This corpus consists of 75 recordings of meetings. Each meeting is recorded with both table-top microphones and close-talking microphones. We have used the table-top microphones, beam-formed into a single channel. SRI has provided us with forced-aligned annotations of the meetings in order to obtain a reference truth for the diarization. Unfortunately, because these alignments were generated for the purpose of training phone models, the utterances considered less useful (e.g., read digit sequences) were not processed and hence these segments were not available in the forced-aligned reference. We have selected the 15 recordings of which most of the utterances were still present in the reference. Speakers occur in multiple recordings in various combinations.

In order to perform query-by-example speaker retrieval, we randomly pick timestamps that contain speech as queries. The task of speaker retrieval is to find all 'documents' in which the speaker at the query-timestamp occurs. The collection is divided into 'documents' of two minutes long each. We consider a document relevant when the query speaker is talking in the document; he or she does not need to be talking the most.

\footnotetext{
${ }^{1}$ Although this is essentially clustering, to avoid confusion with the term clustering for diarization, we will refer to this process as chunk linking
}

The retrieval result is generated from the large scale diarization segmentation as follows. First, the cluster of the speaker that is talking at the query timestamp is determined. Next, all documents are listed in which the speaker is present, sorted by decreasing speaking time. The retrieval result is evaluated using the reference speaker segmentation in a similar fashion. Using the reference segmentation, for each query it is determined who was talking and the list of documents is created in which that speaker is talking as well. With this reference list of relevant documents the Average Precision (AP) and the precision-at-ten of each retrieval result is calculated. The Mean Average Precision (MAP) and the mean precision-at-ten is calculated for the entire set of queries.

The document size was chosen somewhat arbitrarily. For very short documents (less than a second each), average precision is essentially the same as one minus the diarization error rate and very long documents would be impractical for the user to skim through. Two minute long documents could already be considered to be too long to skim through and therefore we also evaluated the system using documents of only 12 seconds (one tenth of two minutes). In total there are 453 and 4530 documents in the archive for document lengths of 2 minutes and twelve seconds, respectively.

Because the queries are generated randomly, speakers that talk a lot in the collection will be queried for more often than others and therefore the MAP will be biased towards these speakers. It depends on the application if this is representative. We also calculated MAP for all queries of each individual speaker and then calculated the total average MAP, a speaker time normalized mean average precision.

Table 1: Speaker retrieval on the ICSI meetings.

\begin{tabular}{|l|c|c|}
\hline Experiment & MAP & P@10 \\
\hline 2min, no normalization & 0.57 & 0.74 \\
2min, speaker normalization & 0.37 & 0.49 \\
12s, no normalization & 0.49 & 0.70 \\
12s, speaker normalization & 0.31 & 0.49 \\
\hline
\end{tabular}

The results of the information retrieval experiments are listed in table 1. In total 10.000 queries were generated and tested. The collection contains 25 speakers. Analysis of the results of individual speakers showed that, with the exception of two speakers, all speakers that occur in less than 50 documents (and speak little in these documents) score with a MAP under 0.3 while only two speakers that occur in more than 50 documents score under 0.3 (figure 2). Speakers that talk little and infrequently are often missed by the diarization system (indirectly because this is not penalized in the NIST diarization evaluation measure, the DER), this explains why these speakers score low on average precision.

\section{TOWARDS LARGE ARCHIVES}

With the experiment from the previous section we showed that the quality of the large scale diarization system is high enough to perform speaker retrieval with promising results. From an information retrieval point of view though the ICSI collection on which we performed this initial speaker retrieval experiments is tiny. To test if our large scale diarization approach is feasible for more realistic applications, we recorded our own archive of Dutch broadcast television.

Because of the size of this archive, during our first attempt to perform large scale diarization on the archive, we faced a number of new challenges. In this section we will describe 


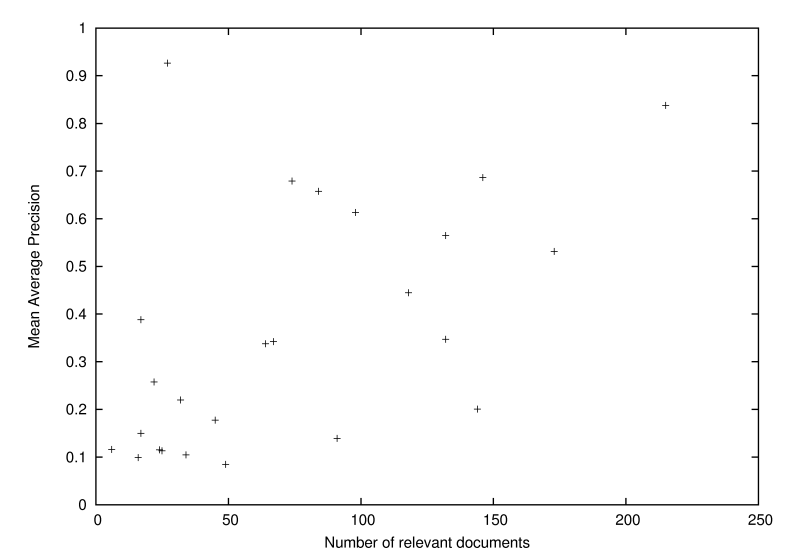

Figure 2: The mean average precision of each speaker in the ICSI meeting collection (every speaker is assigned multiple queries) plotted against the number of relevant documents for each speaker.

these challenges that we feel are especially relevant to large archives. In Section 5 we will explain how we attempted to solve the new challenges encountered for our speaker retrieval system.

\subsection{Dutch television broadcasts}

For over three months (90 days) we have recorded the three public-service Dutch television broadcast channels from 17:30 until midnight. In total we have recorded 1755 hours of video. We also recorded teletext program guide information and teletext subtitles where available. Although 1755 hours of video still might not be very much from an information retrieval perspective, it is considerably more data than the 15.2 hours of audio from the ICSI corpus.

When we tried to apply the large scale speaker diarization approach described in section 3 on the Dutch television broadcasts, we ran into three challenges that we will discuss below.

\subsection{Scaling challenge}

The first four steps of our large scale speaker diarization procedure (see figure 1) scaled perfectly fine to the large data set. Every night directly after the recording of the three channels is finished, each channel is processed by a separate computer. On average this process takes four hours in total (0.62 times real-time). Regarding the first steps of the procedure, processing the data is achieved in linear time. This means that if the archive grows linearly in time (currently 19.5 hours per day), it will always be possible to process the additional recordings in 0.62 times this additional time.

Speech activity detection is the first step in the procedure. From the 1755 hours of audio, the component classified 1083 hours as speech, 291 hours as silence and 381 hours as audible non-speech. In the second step, the 1083 hours of speech is divided into 4204 chunks. Diarization is then performed in the third step on each of these chunks. In total the diarization component generated 58453 speaker clusters that where all processed successfully by the speaker enrollment component.

The scaling challenge starts at scoring each diarization cluster on the models of all other clusters in the archive. The complexity of computing the full scoring matrix is quadratic to the number of clusters, and thus $\mathcal{O}\left(T^{2}\right)$ in size of the archive $T$. In our current implementation, for our archive of 270 channel recordings (90 days), the scoring takes 607.5 hours which is 0.35 times realtime. Our speaker recognition system uses a form of linear scoring [4], which means that a single score of fitting a cluster's speech to another speaker cluster's model is a inner product of a vector of length 26624. The full score matrix can be computed as a matrix-matrix multiplication, for which efficient software libraries exist that fully utilize (parallel) hardware. The dayto-day incremental computation complexity scales $\mathcal{O}(T)$ in the current archive size $T$, where only a stable mount of new clusters models needs to be trained and the cluster speech data needs to be scored to all available cluster models. In total the system requires $0.97 \mathrm{CPU}$ hours $(0.62+0.35)$ to process an additional broadcast hour. Note that it is possible to perform most calculations on parallel processors to reduce the required time.

This calculation is without the actual linking of the speaker clusters (step five of the procedure in figure 1). Performing agglomerative clustering on all the speaker scores (see section 3.1) has an even higher complexity $O\left(T^{3}\right)$ and in our current approach the clustering would need to be repeated for the entire archive each single day. Because of the high complexity and the challenge in speaker distribution that we will discuss below, we decided not to perform the actual speaker linking step in our revised algorithm (see section 5).

\subsection{Challenge in speaker distribution}

The selection from the ICSI corpus has a fairly homogeneous speaker distribution. Not all speakers participate in all meetings, but there is a high degree of overlap which makes linking the speaker clusters easier. Because each speaker occurs in multiple meetings, it is possible to determine at least a few correct links for each speaker cluster. With a balanced set of linked clusters, it is less hard to decide how to link the last few difficult speaker clusters and to decide when to stop clustering.

The broadcast television archive has a much more skewed speaker distribution. It contains a lot more speakers of which some occur regularly in the archive (news anchor persons) and some only once or twice. Even the frequently occurring speakers probably only participate in part of the archive. A game host might appear on television daily, but only during his own time slot. The huge number of speakers and the strongly skewed distribution makes it a lot more challenging to perform the linking step from our large scale diarization procedure. In the following section we will discuss how we changed our procedure to solve this problem.

\subsection{Evaluation challenge}

In order to improve our large scale diarization approach, it needs to be possible to evaluate the system. Evaluation on the ICSI corpus (see 3.1) is possible because reference annotations of the exact time segments that each speaker is talking are available, but creating such reference transcripts for all speech in existing archives is very costly. This means that it is not possible to perform evaluation on the diarization quality (diarization error rate) and also evaluating using recall or the mean average precision, for which all relevant documents of each speaker needs to be known, is not possible.

For the Dutch broadcast television archive, for each program episode we do have a small description available that sometimes contains information about the guests present in 
the episode. We store this information with the intention to use it for automatically labeling the speaker clusters in future research, but it could also be used as a coarse way to evaluate the system: there should be a high overlap between the occurrences of persons in our meta-data and the speaker cluster links.

Alternatively, the result of our system could be evaluated manually. This can only be carried out on a small set of queries (not 10.000 as we did for the ICSI corpus) and only by measuring the precision at the top few documents of the result. In the next section we will present such an experiment on the Dutch broadcast television archive. We will define a number of queries and manually determine the precision-at-ten ( $\mathrm{P} @ 10)$ for each query so that we can calculate the average precision-at-ten.

\section{SPEAKER RETRIEVAL}

In the previous section we have discussed the challenges that we faced during the development of the speaker retrieval system for a Dutch broadcast television archive. Next, we will describe our first attempt in solving these issues. First we will discuss the setup of the system evaluation. Next, we will outline our new procedure and finally we will discuss the evaluation results.

\subsection{Evaluation setup}

As discussed in section 4.4, evaluating a speaker retrieval system for a large archive is very time consuming. For this first small evaluation we will only measure the precisionat-ten (P@10) of a modest set of 30 queries. Identical to the evaluation of the ICSI corpus, we perform query-byexample retrieval. The documents are created by cutting up all material in 12 second pieces and a document is regarded relevant when the query speaker is talking in the document, even for a short while (see section 3.2).

The queries are selected manually from the last day of recordings. We tried to select timestamps both from people that we expect to occur often in the archive (17 queries) and from people that will probably not occur often (13 queries), based on our subjective familiarity with the speakers. Also we selected timestamps from different types of television programs (broadcast news to a game show for children).

We evaluated the system output by watching the top ten documents of each query. We only judged a document relevant if we were $100 \%$ sure that the target speaker was talking (sometimes, e.g., with voice-overs, it is hard to tell).

\subsection{Procedure}

The first steps of the original large scale diarization procedure could be scaled to the larger archive. As before, we first perform speech activity detection, we then divide all speech into chunks and perform speaker diarization on each chunk. Next, we enroll speaker recognition models for each speaker cluster and score the data of each speaker cluster against all speaker models. As calculated in section 4.2, this last procedure is computationally too expensive for very large archives, but for our archive scoring is still relatively straightforward.

Because of the challenge in speaker distribution (see section 4.3), we do not attempt to link all clusters after scoring. Instead, for retrieval we apply the procedure depicted in figure 3. First, the speaker cluster is selected that represents the query timestamp. Next, the score list that was gener- ated by the speaker recognition model that belongs to the speaker cluster is selected. This list contains the scores of all speaker clusters on the query model. The top scores are selected to create the final result list with small documents. Note that because the documents are only 12 seconds long, each speaker cluster will most likely contain multiple documents.

Because we are only able to judge the top ten documents, it is not fair to list all documents from the top ranking clusters. This way only the first or perhaps first two speaker clusters would be evaluated. Therefore, we decided to output only one document for each speaker cluster: the document that contains most speech from the selected speaker cluster.

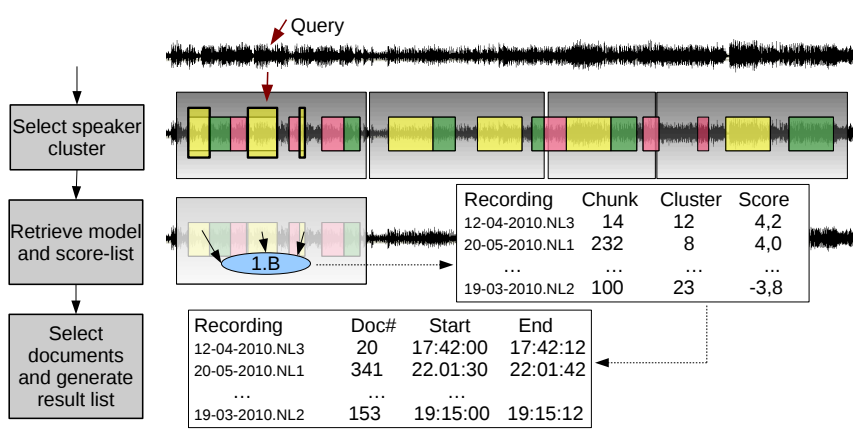

Figure 3: The new retrieval procedure.

\subsection{Evaluation results}

The results of the speaker retrieval evaluation are listed in table 5.3. The average precision-at-ten for this evaluation is 0.52 . As expected because of the increased complexity of the task, this score is lower than the average precision-at-ten of 0.70 from the ICSI meeting corpus evaluation. Although we realize that for a proper evaluation, a lot more queries are needed and that recall should be calculated as well, this result at the least indicates that the output of the speaker retrieval system is not random.

As expected, the $\mathrm{P} @ 10$ of frequently occurring speakers is higher than the $\mathrm{P} @ 10$ of the other speakers. For less frequently occurring speakers this might even be because they do not occur even ten times in the archive.

We also noticed that the precision-at-ten is significantly lower than average for queries that point to impure speaker clusters. Such an impure cluster might not be very representative for the query when a) the target speaker is misclassified by the diarization component or b) when the speaker cluster is polluted by music or sound-effects. Typically, the diarization component will misclassify a speaker when he or she is only talking for a very short period of time of the chunk. This was the case for example when a short fragment of a politician (Balkenende, the Dutch prime minister) is quoted in broadcast news. Although this person could be retrieved with a $\mathrm{P} @ 10$ of 1.0 in another query, the result of this particular query was only 0.3. In other cases, the speaker cluster was so much polluted by audible non-speech, that in fact not the target speaker was retrieved, but various other sound effects.

If the speech activity detection component falsely classifies speech as non-speech, it is possible that no speaker cluster is available for a particular query. This happened for one of the thirty queries. 
Table 2: Speaker retrieval results (P@10) of our television broadcast evaluation.

\begin{tabular}{|l|c|c|}
\hline Description & \#queries & P@10 \\
\hline Total evaluation set & 30 & 0.520 \\
\hline Frequently occurring people & 17 & 0.800 \\
Less frequently occurring people & 13 & 0.154 \\
\hline Query represents clean cluster & 18 & 0.756 \\
Query represents polluted cluster & 12 & 0.167 \\
\hline
\end{tabular}

\section{DISCUSSION AND FUTURE WORK}

In this paper we have shown that it is possible to perform speaker retrieval, retrieve audiovisual fragments from an archive in which a particular person is talking, by combining speaker diarization and speaker recognition techniques. We have first evaluated our speaker retrieval system on a relatively small collection of meeting recordings and next we have performed a modest evaluation on a much larger multimedia archive.

Our first attempt to adjust our system for this larger archive revealed a number of challenges. Scaling the scoring procedure of the speaker recognition models proved to be possible for the large archive we tested on, but it will be computationally expensive to use this method for even larger archives. We hope to solve this challenge in future work by using a different clustering approach, e.g., by dividing speakers in smaller classes beforehand (for example based on gender) so that not all speakers need to be scored against each other. If we are able to identify frequently occurring speakers automatically, e.g., anchor persons, reporters, celebrities, and make a single model for these, the number of models to score against may stay limited.

Another challenge was the fact that the large archive contains a lot of speakers that do not necessarily occur frequently in the archive. This makes linking the speaker clusters significantly more complicated. We solved this problem in our new retrieval approach by using the speaker recognition scoring lists directly for each query instead of making a hard decision for every speech fragment beforehand. This works well for retrieval tasks, but it needs to be investigated if it is possible to use this approach for other applications such as for example meeting assistants, where hard decisions on the identity of each speaker are needed.

The evaluation of the system for the large archive was limited due to a lack of reference transcriptions. For smaller collections it is affordable to annotate reference transcriptions, but for large archives being able to evaluate becomes an issue. Even though the evaluation was limited, we discovered that the precision of our system is highly depending on the quality of the speaker model picked for ranking the document list. A diarization classification error in one of the documents that the query speaker occurs in will just result in one missing document. A diarization error of the segment that is being used as query will result in missing all documents. In future research we will investigate if we can reduce this risk by using the approach mentioned earlier, iteratively merging speaker clusters of the same speaker and training more robust models for these speakers.

Another way of reducing errors made by misclassification of the query speaker is to develop a system that provides feedback to the user about the choice of his query timestamp. For example, we could show keyframes of other speech segments from the selected speaker cluster. If these keyframes are from other speakers or obviously not from a speaker at all, the user is able to pick another timestamp as query. Obviously, this approach will not be possible for audio archives.

In future work we will investigate extending our system with automatically obtained speaker labels (names, age, etc). We might be able to do this by using external information sources such as guide information, subtitles or automatic speech recognition output.

\section{ACKNOWLEDGMENTS}

This paper is based on research carried out for the BATS project within the research program IM-Pact funded by IBBT and ICTRegie.

\section{REFERENCES}

[1] R. B. Dunn, D. A. Reynolds, and T. F. Quatieri. Approaches to speaker detection and tracking in conversational speech,. Digital Signal Processing, 10(1-3):93 - 112, 2000.

[2] A. J. et all. The ICSI meeting project: Resources and research. In NIST ICASSP 2004 Meeting Recognition Workshop, Montreal, May 2004.

[3] J. G. Fiscus, J. Ajot, and J. S. Garofolo. The rich transcription 2007 meeting recognition evaluation. In Multimodal Technologies for Perception of Humans, Lecture Notes in Computer Science, Berlin, 2008.

[4] O. Glembek, L. Burget, N. Dehak, N. Brümmer, and P. Kenny. Comparison of scoring methods used in speaker recognition with joint factor analysis. In Proc ICASSP 2009, Taipei, Taiwan, April 2009.

[5] M. Huijbregts and D. van Leeuwen. The RU submission to the Evalita'09 "application track" speaker recognition evaluation. In proceedings of Evalita 2009, 2009.

[6] M. Huijbregts and D. van Leeuwen. Large scale speaker diarization for long recordings and small collections. IEEE Transactions on Audio, Speech and Language Processing, submitted.

[7] M. Huijbregts, C. Wooters, and R. Ordelman. Filtering the unknown: Speech activity detection in heterogeneous video collections. In proceedings of Interspeech, Antwerp, Belgium, August 2007.

[8] A. Martin and M. Przybocki. The nist 1999 speaker recognition evaluation-an overview. Digital Signal Processing, 10(1-3):1 - 18, 2000.

[9] D. Reynolds and P. Torres-Carrasquillo. Approaches and applications of audio diarization. pages 953-956, Philadelphia, PA, March 2005.

[10] L. Rodríguez, M. Penagarikano, and G. Bordel. A simple but effective approach to speaker tracking in broadcast news. Lecture Notes in Computer Science, 2007.

[11] D. van Leeuwen and M. Huijbregts. The AMI speaker diarization system for NIST RT06s meeting data. In (MLMI), volume 4299 of Lecture Notes in Computer Science, pages 371-384, Berlin, October 2007.

[12] C. Wooters and M. Huijbregts. The ICSI RT07s speaker diarization system. In Multimodal Technologies for Perception of Humans, Lecture Notes in Computer Science, Berlin, 2008. Springer Verlag.

[13] J. Zibert, B. Vesnicer, and F. Mihelic. A system for speaker detection and tracking in audio broadcast news. Informatica (Slovenia), 32(1):51-61, 2008. 